\title{
O PAPEL EDUCATIVO DA EQUIPE DE ENFERMAGEM COMO MEIO DE GARANTIR AOS PACIENTES PORTADORES DE HIPERTENSÃO ARTERIAL SISTÊMICA A ADESÃO AO TRATAMENTO MEDICAMENTOSO E NÃO-MEDICAMENTOSO
}

\author{
Kethley Karoline Furtado da Silva de Souza ${ }^{1}$ \\ Érika de Fátima Magno Machado ${ }^{2}$ \\ Kyssila Daineze Pinto da Silva 3 \\ Basílio Magno Francisco Rodrigues da Silva 4 \\ Ely de Almeida Glória Filho 5
}

RESUMO: A hipertensão arterial (HA) é um grave fator de risco para o desenvolvimento de doenças cardiovasculares, caracterizando-se como importante doença na área da saúde pública. O tratamento da $\mathrm{HA}$ consiste na terapia medicamentosa e tratamento nãomedicamentoso ou mudança no estilo de vida. Teve por objetivo apresentar a importância da equipe de enfermagem na aderência ao tratamento por parte dos pacientes portadores de HAS. Trata-se de um estudo qualitativo, de revisão sistemática da literatura dos últimos anos. A educação em saúde adequada para os pacientes hipertensos garante aos pacientes maiores chances de aderirem ao tratamento e ter consequente redução de riscos.

Palavras-chave: Educação em enfermagem. Adesão. Tratamento medicamentoso.

ABSTRACT: Hypertension $(\mathrm{AH})$ is a serious risk factor for the development of cardiovascular diseases, characterizing it as an important disease in the public health area. HA treatment consists of drug therapy and non-drug treatment or lifestyle change. It aimed to present the importance of the nursing staff with adherence to treatment by patients with hypertension. This is a qualitative study, a systematic review of the literature of recent years. Proper health education for hypertensive patients ensures that patients are more likely to adhere to treatment and consequently reduce risks.

Keywords: Adherence. Nursing education. Drug treatment.

\section{INTRODUÇÃO}

A hipertensão arterial (HA) é uma síndrome ocasionada por múltiplos fatores e constitui uma das maiores questões de saúde pública e tem sido reconhecida como um grave agente de risco para o desenvolvimento de doenças cardiovasculares.

\footnotetext{
${ }^{I}$ Bacharelado em Enfermagem - Centro Universitário Redentor.

${ }^{2}$ Bacharelado em Enfermagem - Centro Universitário Redentor.

${ }^{3}$ Bacharelado em Enfermagem - Centro Universitário Redentor.

${ }^{4}$ Bacharelado em Enfermagem - Centro Universitário Redentor.

${ }^{5}$ Bacharelado em Enfermagem - Centro Universitário Redentor.
} 
Se caracteriza como uma doença sistêmica que envolve modificações na parede das artérias e do miocárdio comparticipante à disfunção endotelial e constrição e reformulamento da musculatura lisa vascular. A hipertensão arterial hoje é definida de acordo com valores pressóricos, nas quais níveis iguais ou maiores que $130 / 80 \mathrm{mmHg}$, observados em duas ou mais aferições da pressão arterial, caracterizam a doença. (OLIVEIRA, 2OII ; AMERICAN HEART ASSOCIATION, 2017)

Um problema relacionado a HA é que, na maioria dos casos, apresenta ausência de sintomas, sendo manifesta apenas quando há comprometimento de algum órgão. (MENEZES e GOBBI,2010)

Considerada uma doença de natureza crônica, a hipertensão não é uma doença transmissível e pode ser controlada, mas não é curável; fazendo com que o paciente seja tratado por toda a vida. (VITOR et al, 20II)

Existem questões que contribuem com a elevação da pressão arterial e consequente desenvolvimento de doenças cardiovasculares, como: Alimentação rica em sódio e gorduras, ausência de exercício físico regular, tabagismo, etilismo, além de alterações psicoemocionais. (MIO JR,2002)

Mudanças no estilo de vida que inclua o controle de peso, a redução da ingestão excessiva de álcool e sal, extinção do habito de fumar, além do desenvolvimento de práticas de atividade física são fatores relacionados a prevenção primaria da elevação da pressão arterial. (OLIVEIRA, 20II)

A abordagem terapêutica inclui o tratamento medicamentoso e nãomedicamentoso. Para garantir a adesão ao tratamento é necessário que o paciente reconheça a doença e esteja disposto a aceitar o tratamento, além de seguir todas as orientações; Esse processo é importante para que haja um autocuidado por parte do próprio paciente frente aos fatores de risco. (PINOTTI et al, 2008)

Dentre os fatores que dificultam a adesão ao tratamento, a falta de informações sobre a doença é um agravante. (RIBEIRO et al,20I2)

Sendo assim, faz-se necessário o envolvimento de uma equipe multidisciplinar bem orientada sobre a doença, suas características e formas de tratamento. Uma equipe que estea pronta para fornecer todo o tipo de orientação necessária ao portador da doença. 
O enfermeiro como parte da equipe de saúde, assume parte da responsabilidade de cuidado para a promoção da saúde, prevenção de riscos e agravos da doença e também acompanha o portador da doença. Com seu conhecimento cientifico e de educador ele instrui o paciente para o tratamento melhorando sua qualidade de vida e o tornando corresponsáveis pelo processo saúde-doença. (VITOR et al ,20II)

\section{QUESTÃO NORTEADORA}

De que forma o papel educativo da equipe de enfermagem garante aos pacientes portadores de HAS a adesão ao tratamento medicamentoso e não-medicamentoso?

\section{${ }_{3}$ OBJETIVOS}

\section{I OBJETIVO GERAL}

Apresentar a importância da equipe de enfermagem na aderência ao tratamento por parte dos pacientes portadores de HAS.

\subsection{OBJETIVOS ESPECÍFICOS}

- Caracterizar a HAS;

- Registrar as formas de tratamento ;

- Analisar os fatores que dificultam o comprometimento dos pacientes ao tratamento;

- Identificar qual deve ser a abordagem da equipe de enfermagem frente aos fatores que dificultam o comprometimento dos pacientes com o tratamento;

\section{JUSTIFICATIVA}

Ao constatar um baixo índice da anuência ao tratamento da hipertensão arterial sistêmica por parte dos pacientes, ficou claro que seria viável analisar o tema em questão, levando em consideração que esta patologia representa um grande problema de saúde pública. Essa questão motivou os autores, estudantes do curso de enfermagem, a investigarem o que levara a esta situação .

A pesquisa leva ao fato de que, o que ocasiona esse fator é a falta de conhecimento por parte dos paciente de sua patologia e o quão importante é a anuência ao tratamento . 
O trabalho traz o alerta a necessidade de educação desses pacientes quanto a sua doença; ressalta também a importância da equipe de enfermagem como cumpridora desse papel e aponta ao fato de que a execução deste é fundamental para aceitação do tratamento pelos pacientes.

\section{METODOLOGIA}

O presente estudo será baseado em referências bibliográficas pertinentes ao assunto em questão, que nortearão a pesquisa com fundamentos e conhecimentos para o trabalho acadêmico. Utilizamos como base artigos científicos e revisões bibliográficas.

Para a elaboração deste estudo, serão consultados periódicos indexados ao LILACS e SCIELO e BVSALUD, através de uma pesquisa bibliográfica de artigos científicos publicados sobre a temática da educação em saúde da enfermagem quanto aos pacientes hipertensivo.

Para isto, priorizamos as publicações acerca do tema encontradas nos periódicos a partir do ano 2000 . Para fins de estudo, foram consideradas as publicações relacionadas ao cuidado de enfermagem para com os pacientes que sofrem de hipertensão arterial sistêmica

, utilizando como palavras-chaves: "educação em enfermagem”, "adesão" , "não-adesão" “tratamento medicamentoso" e "tratamento não-medicamentoso".

A escolha dos artigos foi realizada mediante a leitura dos resumos, a fim de confirmar a temática proposta. Tais resumos demonstravam estudos de caso e referências bibliográficas que abordavam a educação em saúde da enfermagem e como ela pode atuar dentro deste contexto proposto por tal estudo, que se trata de uma revisão bibliográfica.

\section{REFERENCIAL TEÓRICO}

\section{I HIPERTENSÃO ARTERIAL SISTÊMICA}

A pressão arterial é a pressão existente no interior das artérias, comunicada às suas paredes e quaisquer fatores que que interferem nestes pode alterar a PA. (OIGMAN, 1987)

A hipertensão arterial é caraterizada pela elevação da PA, é uma doença crônica persistente, de elevado custo econômico-social, principalmente em decorrência das suas complicações, como por exemplo o acidente vascular cerebral e o infarto de miocárdio com grande impacto na morbimortalidade brasileira e do mundo. Existem alguns fatores, 
considerados fatores de risco que, associados entre si e a outras condições, favorecem o aparecimento da hipertensão arterial, sendo: idade, sexo, antecedentes familiares, raça, obesidade, estresse, vida sedentária, álcool, tabaco, anticoncepcionais, alimentação rica em sódio e gorduras. (MACMOHAN et al,1995) (CHOBANIAN,2003)

O diagnóstico de hipertensão arterial é baseado num procedimento relativamente simples, a medida da pressão arterial, envolvendo a grande responsabilidade de decidir se um paciente é normotenso ou hipertenso. As consequências de um diagnóstico errôneo são desastrosas; o diagnóstico de normotensão, num hipertenso, irá privá-lo dos benefícios do tratamento, ao passo que o de hipertensão, num normotenso, irá submetê-lo aos malefícios do tratamento desnecessário. (MION JR et al, 1996).

A hipertensão arterial pode ser classificada conforme níveis tensionais obtidos (Quadro I).

Quadro I - Classificação da Hipertensão arterial sistêmica

\begin{tabular}{|c|c|c|c|}
\hline BLOOD PRESSURE CATEGORY & $\begin{array}{c}\text { SYSTOLIC mm Hg } \\
\text { (upper number) }\end{array}$ & $\begin{array}{c}\text { DIASTOLIC mm Hg } \\
\text { (lower number) }\end{array}$ \\
\hline NORMAL & LESS THAN 120 & and & LESS THAN 80 \\
\hline ELEVATED & $120-129$ & and & $80-89$ \\
\hline $\begin{array}{c}\text { HIGH BLOOD PRESSURE } \\
\text { (HYPERTENSION) STAGE 1 }\end{array}$ & $130-139$ & or \\
\hline $\begin{array}{c}\text { HIGH BLOOD PRESSURE } \\
\text { (HYPERTENSION) STAGE 2 }\end{array}$ & 140 OR HIGHER & or & 90 OR HIGHER \\
\hline $\begin{array}{c}\text { HYPERTENSIVE CRISIS } \\
\text { (consult your doctor immediately) }\end{array}$ & HIGHER THAN 180 & and/or & HIGHER THAN 120 \\
\hline
\end{tabular}

Fonte : American Heart Association (2017)

A HAS também pode ser classificada, também, de acordo com sua causa base conforme descrito na Linha Guia de hipertensão arterial do Paraná (2014,p.26)

A maioria dos casos de hipertensão arterial não apresenta uma causa aparente facilmente identificável, sendo conhecida como hipertensão essencial. Uma pequena proporção dos casos de hipertensão arterial é devida a causas muito bem estabelecidas, que precisam ser devidamente diagnosticadas, uma vez que, com a remoção do agente etiológico, é possível controlar ou curar a hipertensão arterial. É a chamada hipertensão secundária. No nível de Atenção Básica, a equipe de saúde deve estar preparada para diagnosticar, orientar e tratar os casos de hipertensão essencial, que são a maioria. Por outro lado, os casos suspeitos de hipertensão secundária deverão ser encaminhados a especialistas. 


\subsection{FORMAS DE TRATAMENTO}

O tratamento da HAS consiste no tratamento medicamentoso e nãomedicamentoso.

\subsection{TRATAMENTO MEDICAMENTOSO}

O tratamento medicamentoso anti-hipertensivo deve apresentar eficácia via oral, ser bem tolerado pelo paciente e possibilitar a administração no menor número de vezes ao dia, de preferência em dose única.(BRASIL,20o6)

Segundo o III Consenso de Hipertensão arterial (1998)

Os medicamentos preferenciais para o controle da pressão arterial em monoterapia inicial são diuréticos, betabloqueadores, antagonistas dos canais de cálcio, inibidores da enzima conversora da angiotensina e antagonistas do receptor da angiotensina II.

O tratamento deve ser individualizado e a escolha inicial do medicamento como monoterapia deve basear-se no mecanismo fisiopatogônico predominante, nas características individuais, nas doenças associadas, nas condições socioeconômicas e na capacidade de o medicamento influir sobre a morbidade e a mortalidade cardiovasculares.

A dose do medicamento como monoterapia deve ser ajustada até que se consiga redução da pressão arterial a um nível considerado satisfatório para cada paciente (em geral, interior a $140 / 90 \mathrm{mmHg}$ ). $\mathrm{O}$ ajuste deve ser feito buscando-se a menor dose eficaz, ou até que surjam efeitos indesejáveis. Se o objetivo terapêutico não for conseguido com a monoterapia inicial, são possíveis três condutas:

a) Se o efeito for parcial ou nulo e sem reação adversa, recomendase o aumento da dose do medicamento.

b) Quando não ocorrer efeito na dose máxima pré-conizada, ou se surgirem efeitos indesejáveis, recomenda-se a substituição da droga em monoterapia. 
c) Se, ainda assim, a resposta for inadequada, devem-se associar duas ou mais drogas.

Quadro 2-Classes de anti-hipertensivos mais comumente disponíveis nas Unidades de Atenção Básica

\begin{tabular}{|c|c|c|}
\hline Classe & Mecanismo de ação & $\begin{array}{l}\text { Principais } \\
\text { representantes }\end{array}$ \\
\hline Diuréticos & $\begin{array}{l}\text { O mecanismo de ação anti-hipertensiva dos diuréticos relaciona- } \\
\text { se inicialmente aos seus efeitos diuréticos e natriuréticos, com } \\
\text { diminuição do volume extracelular. Posteriormente, após cerca } \\
\text { de quatro a seis semanas, o volume circulante praticamente se } \\
\text { normaliza e há redução da resistência vascular periférica. São } \\
\text { eficazes no tratamento da hipertensão arterial, tendo sido } \\
\text { comprovada sua eficácia na redução da morbidade e da } \\
\text { mortalidade cardiovasculares. Para uso como anti-hipertensivos, } \\
\text { são preferidos os diuréticos tiazídicos e similares, em baixas } \\
\text { doses. A espironolactona é poupador de potássio, por isso, seu } \\
\text { uso exige cuidados especiais. }\end{array}$ & $\begin{array}{l}\text { Hidroclorotiazida } \\
\text { Clortalidona } \\
\text { Idapamina } \\
\text { Furosemida } \\
\text { Espironolactona }\end{array}$ \\
\hline $\begin{array}{l}\text { Inibidores } \\
\text { adrenérgicos de } \\
\text { ação central }\end{array}$ & $\begin{array}{l}\text { Agonistas alfa- } 2 \text { centrais: atuam estimulando os receptores alfa- } \\
2 \text { adrenérgicos pré-sinápticos no sistema nervoso central, } \\
\text { reduzindo o tônus simpático. }\end{array}$ & $\begin{array}{l}\text { Alfametildopa } \\
\text { Clonidina }\end{array}$ \\
\hline $\begin{array}{l}\text { Beta-bloqueadores } \\
\text { (BB }\end{array}$ & $\begin{array}{l}\text { Bloqueadores beta-adrenérgicos: seu mecanismo anti- } \\
\text { hipertensivo envolve diminuição inicial do débito cardíaco, } \\
\text { redução da secreção de renina, readaptação } \\
\text { dos barorreceptores e diminuição das catecolaminas nas } \\
\text { sinapses nervosas. }\end{array}$ & $\begin{array}{l}\text { Propranolol } \\
\text { Atenolol } \\
\text { Carvedilol }\end{array}$ \\
\hline $\begin{array}{l}\text { Bloqueadores dos } \\
\text { canais de cálcio } \\
(\mathrm{BCC})\end{array}$ & $\begin{array}{l}\text { Agem fundamentalmente pela inibição da enzima conversora da } \\
\text { angiotensina (ECA), bloqueando a transformação da } \\
\text { angiotensina I em II no sangue e nos } \\
\text { tecidos, embora outros fatores possam estar envolvidos nesse } \\
\text { mecanismo de ação. São eficazes no tratamento da HAS, } \\
\text { reduzindo a morbidade e a mortalidade cardiovasculares nos } \\
\text { hipertensos. }\end{array}$ & Anlodipina \\
\hline $\begin{array}{l}\text { Inibidores } \\
\text { da enzima } \\
\text { conversora da } \\
\text { angiotensina } \\
\text { (IECA) }\end{array}$ & $\begin{array}{l}\text { Agem fundamentalmente pela inibição da enzima conversora da } \\
\text { angiotensina (ECA), bloqueando a transformação da } \\
\text { angiotensina I em II no sangue e nos } \\
\text { tecidos, embora outros fatores possam estar envolvidos nesse } \\
\text { mecanismo de ação. São eficazes no tratamento da HAS, } \\
\text { reduzindo a morbidade e a mortalidade cardiovasculares nos } \\
\text { hipertensos. }\end{array}$ & $\begin{array}{l}\text { Captopril } \\
\text { Enalapril }\end{array}$ \\
\hline $\begin{array}{l}\text { Bloqueadores } \\
\text { do receptor AT1 } \\
\text { da angiotensina II } \\
\text { (ARA II) }\end{array}$ & $\begin{array}{l}\text { Antagonizam a ação da angiotensina II por meio do bloqueio } \\
\text { específico de seus receptores AT1. }\end{array}$ & Losartana \\
\hline
\end{tabular}

Fonte: Adaptado de Sociedade Brasileira de Cardiologia (20ro)

Vale ressaltar que a hipertensão é uma doença crônica e exige tratamento continuado por toda vida, ou seja, não se deve deixar de tomar o medicamento por perceber uma "melhora" no quadro da doença, já que a mesma apresenta momentos de silêncio. O uso não pode ser suspenso sem orientação médica 
caso não se perceba melhoria no quadro já que é de suma importância que o tratamento esteja associado a ao tratamento não-medicamentoso.

\subsubsection{TRATAMENTO NÃO-MEDICAMENTOSO}

O tratamento não-medicamentoso da HAS se objetiva a diminuir a morbidade e a mortalidade cardiovasculares por meio de mudanças do estilo de vida que favoreçam a redução da pressão arterial. (OLIVEIRA, 2oII)

As mudanças no estilo de vida estão indicadas a todos os pacientes e são eficazes em reduzir a pressão arterial, desde que realizadas adequadamente.(LINHA GUIA DE HIPERTENSÃO-PR ,2014 p.29)

As medidas comprovadas que reduzem a pressa o arterial são: redução do peso corporal, da ingestão do sal e do consumo de bebidas alcoólicas, prática de exercícios físicos com regularidade, e a não-utilização de drogas que elevam a pressão arterial. Essas medidas apresentam custo reduzido e baixo risco de complicações, associada ao tratamento medicamentoso aumenta a sua eficácia.(MIO JR, 2002).

\section{REDUÇÃO OU CONTROLE DE PESO}

Segundo a Linha Guia de Hipertensão do Paraná (2014 p.30)

O excesso do peso é um fator de risco para a hipertensão arterial sistêmica, a dislipidemia e a doença cardiovascular. Estima-se que $20 \%$ a $30 \%$ da prevalência da hipertensão pode ser explicada pela presença do excesso de peso. A redução de 5 a Io\% do peso corporal associa-se à melhora significativa do controle metabólico e dos níveis de PA. O objetivo é alcançar uma perda sustentada e progressiva de 0,5 a I $\mathrm{kg}$ de peso por semana. A meta é alcançar um índice de massa corporal (IMC) inferior a $25 \mathrm{~kg} / \mathrm{m} 2$ e circunferência da cintura inferior a $94 \mathrm{~cm}$ para homens e $80 \mathrm{~cm}$ para mulheres, embora a diminuição de $5 \%$ a $10 \%$ do peso corporal inicial já seja capaz de produzir redução da pressão arterial. 
As estratégias para o médico no controle e diminuição do peso do paciente são: a identificação desse índice e da dieta real do hipertenso, a fim de tomar conhecimento do que exatamente ele come e do que modificar; traçar objetivos de peso a curto, médio e longo prazo; orientá-lo a seguir as medidas associadas, explicadas a seguir, e providenciar apoio psicológico. (FERREIRA, 20II).

\section{PADRÃO ALIMENTAR ADEQUADO}

A dieta do hipertenso deverá ser pobre em sal e rica em potássio, magnésio e cálcio. A dieta pobre em sal (hipossódica), deverá restringir a ingestão diária de sal em 6 gramas (2,4 gramas de sódio), ou seja, 4 colheres rasas de café de sal para o preparo dos alimentos (4 gramas de sal), mais 2 gramas de sal próprio dos alimentos (evite: conservas , frios, enlatados, embutidos, molhos prontos, sopas de pacote, queijos amarelos, salgadinhos, etc.)

A dieta hipossódica pode reduzir a pressão arterial sistólica em 2 a $8 \mathrm{mmHg}$. Uma dieta rica em potássio e magnésio poderá ser obtida através de uma ingesta rica de feijões, ervilhas, vegetais verdes escuros, banana, melão, cenoura, beterraba, frutas secas, tomates, batata inglesa e laranja. Uma dieta, chamada de DASH, composta de frutas, verduras, fibras, alimentos integrais, leite desnatado, pobre em colesterol e gorduras saturadas, demonstrou ser capaz de reduzir a pressão arterial sistólica em 8 a $14 \mathrm{mmHg}$. (SOUZA, 20II ; AMODEO,1996)

\section{DIMINUIÇÃO DO CONSUMO DO ÁLCOOL}

A relação entre o alto consumo de bebida alcoólica e a elevação da pressão arterial tem sido relatada em estudos observacionais. (MACMAHON,1987)

Recomenda-se limitar a ingestão de bebida alcoólica a $30 \mathrm{ml} / \mathrm{dia}$ de etanol para homens e a metade dessa quantidade para mulheres. Isso corresponde, para o homem, a aproximadamente $720 \mathrm{ml}$ de cerveja; $240 \mathrm{ml}$ de vinho e $60 \mathrm{ml}$ de bebida destilada. Aos pacientes que não conseguem se enquadrar nesses limites de consumo sugere-se o abandono do consumo de bebidas alcoólicas (KRAUS et al.,200o). 


\section{ABANDONO DO TABAGISMO}

O tabagismo aumenta o risco de complicações cardiovasculares. Portanto, os hipertensos que fumam devem ser repetidamente estimulados a abandonar esse hábito por meio de aconselhamento e medidas terapêuticas de suporte específicas. (FORJAZ,2003 ; BALDISSERA et al., 2009)

\section{PRÁTICA DE ATIVIDADES FÍSICAS}

Pacientes hipertensos devem iniciar programas de exercícios físicos regulares, desde que tenham sido submetidos à avaliação clínica prévia. Além de diminuir a pressão arterial, o exercício físico pode reduzir consideravelmente o risco de doença arterial coronária, acidentes vasculares cerebrais e mortalidade geral (A). Programas de exercícios físicos, para a prevenção primária ou secundária de doenças cardiovasculares, devem contar com atividades aeróbias dinâmicas, tais como caminhadas rápidas, corridas leves, natação e ciclismo. Recomenda-se a freqüência de três a seis vezes por semana, intensidade moderada e sessões de 30 a 60 minutos de duração. Para o controle da intensidade do exercício podem ser utilizados tanto a freqüência cardíaca como o consumo de oxigênio (60\% a $80 \%$ da freqüência cardíaca máxima ou $50 \%$ a $70 \%$ do consumo máximo de oxigênio). (BERLIN e COLDITZ,2009)

\subsection{FATORES QUE DIFICULTAM O COMPROMETIMENTO DOS PACIENTES COM O TRATAMENTO}

São grandes os avanços científicos e tecnológicos que permeiam o tratamento da hipertensão arterial, a grande dificuldade nesse âmbito é justamente a adesão aos tratamentos propostos pela equipe de saúde, por parte dos próprios pacientes. Considera-se adesão ao tratamento o grau de sincronia entre a prescrição médica, que inclui os orientações medicamentosas e não-medicamentosas, e o comportamento do paciente. $\mathrm{Na}$ abordagem da HAS, envolve o uso efetivo do medicamento, realização de mudanças no estilo de vida e comparecimento às consultas médicas. (HORWITZ RI,I993; HORWITZ SM,1993)

A aceitação do paciente à determinada abordagem terapêutica depende de vários aspectos como, dentre outros, relação médico-paciente, às questões subjetivas do paciente, 
às questões referentes ao tratamento, à doença, ao acesso ao serviço de saúde, à obtenção do medicamento prescrito e à continuidade do tratamento. (CHIZZOLA et al.,1996)

Os pacientes podem apresentar também um comportamento infantil, que faz com que ele dependa de uma rede de apoio para diversas questões relacionadas a sua vida, inclusive no que se refere a sua saúde. O paciente pode não se considerar doente por conta da falta de sintomas da doença. (MINAYO,1993)

A adesão ao programa terapêutico também pode ser mais difícil para os idosos, por conta da diminuição da habilidade de aprender e adquirir novos domínios e informações. (SMELTZER e BARE, 2005)

Não se pode negar que o baixo nível socioeconômico e cultural também é um fator que dificulta o tratamento e controle efetivo da pressão arterial que pode ser empecilho no prosseguimento do tratamento. (PIERIN, et al.,200I)

As crenças do indivíduo também ocasionam mudanças em sua vida cotidiana. Muitas pessoas acreditam não ser possível obter melhora em seu quadro com mudanças em seu estilo de vida ,o que faz com que o tratamento não seja valorizado. Por outro lado, temse os pacientes que valorizam mais os remédios caseiros do que os comprimidos e que só buscam os serviços de saúde em caso de piora de seu quadro. (PIRES E MUSSI,2008; MOREIRA et AL.,2009)

\subsection{ABORDAGEM DA EQUIPE DE ENFERMAGEM FRENTE AOS FATORES QUE DIFICULTAM O COMPROMETIMENTO DOS PACIENTES COM O TRATAMENTO}

Evidentemente, a HAS e o seu tratamento adequado envolvem uma multiplicidade de fatores extremamente complexos, que exigem de todos os envolvidos o emprego de estratégias combinadas que dêem conta dessa complexidade. (SCHROEDER et al.,2004)

A atuação de uma equipe de atenção à saúde é importante a fim de orientar, assistir, diagnosticar e tratar o hipertenso, assegurando-lhe controle adequado da pressão arterial. Entre estes profissionais destaca-se o enfermeiro, que representa importante papel nesta assistência. Entre os cuidados que o paciente hipertenso deve observar estão o estilo de vida, que deve ser alterado parar ser mais saudável, a adesão ao tratamento e o controle adequado da pressão arterial. É, portanto um desafio para os profissionais da saúde e dentre 
eles o enfermeiro, cuidar de pacientes hipertenso, especialmente os idosos, assegurando que tenham qualidade de vida adequada. (KIELLER e CUNHA,2014)

O profissional de saúde deve estar preparado para interagir com o paciente levando em conta suas perspectivas, concepções, significações sobre o usuário, sobre a doença (HAS) e o tratamento, de forma a incentivar sua adesão ao tratamento (REINERS e NOGUEIRA,2009)

Há necessidade de elaboração de estratégias para garantir o acesso a tratamentos que possibilitem diminuir o número de medicamentos prescritos e o número de administrações diárias, uma estratégia importante é a oferta pelo Sistema Único de Saúde (SUS) e uma educação eficiente quanto à forma correta de utilizá-los. (TAVARES et al,2013;MOTTER et al.,)

A ESF configura-se como elemento-chave para a organização e implantação de ações que visem ao controle da HAS. Destacam-se a necessidade do trabalho multiprofissional e interdisciplinar para a abordagem ao hipertenso e o cuidado domiciliar como uma estratégia para maior adesão ao tratamento e controle da doença, para, assim, minimizar os seus impactos e os da não adesão tanto para o sistema de saúde quanto para os usuários, a ESF garante maior vínculo entre o paciente e equipe promovendo assim uma relação de confiança. (RIBEIRO et al.,20I2)

Assim, o enfermeiro busca a excelência no atendimento com objetivo de proporcionar um treinamento de forma clara, onde o paciente sinta-se à vontade para esclarecer suas dúvidas e realizar o protocolo de exame da melhor forma possível. $O$ enfermeiro se faz presente em todas as etapas deste processo que se inicia a partir da escolha do aparelho e pendura durante a orientação do manejo deste, do preenchimento do diário de atividades, da realização de exame propriamente dito e finaliza na emissão do relatório. A satisfação do paciente durante esse processo tem relação direta com a maneira de realização do protocolo e uma das formas de assegurar o conforto e segurança durante este processo é a disponibilidade de um contato direto com o profissional de enfermagem. (SMELTZER e BARE,2005)

Galvão et al. (2009 p.4I), a equipe de saúde quando bem articulada, esclarecer o paciente "não apena sobre a doença, mas sobre seu papel o tratamento". Essa ação permite 
que o paciente se envolva com seu tratamento, desenvolvendo condutas que cooperem com seu tratamento.

A orientação de pacientes com hipertensão arterial sistêmica cabe em grande parte ao enfermeiro, uma vez que a função educativa faz parte de suas atribuições, a qual for redimensionada pelo contato constante junto ao paciente. Sendo assim, Kieller e Cunha (2004) afirmam que o desenvolvimento da relação entre o profissional de saúde e o paciente é estimulado criando desse modo o vínculo pessoal com intuito de incentivar mudanças radicais e profundas nos hábitos de vida desse paciente.

\section{CONSIDERAÇÕES FINAIS}

Embora a hipertensão arterial sistêmica seja uma doença bastante conhecida, de grande prevalência mundial e fonte de inúmeros agravos cardiovasculares, ainda existem inúmeras questões a serem trabalhadas no que tange ao seu tratamento.

Com o estudo em questão percebe-se um elevado índice de falta de adesão ao tratamento proposto, tanto medicamentoso quanto não-medicamentoso. Dentre diversos outros fatores, o mais preocupante é a falta de informação que esses pacientes recebem por parte da equipe de saúde o que faz com que eles não se comprometam com o tratamento.

Diante desse fato, faz-se necessário a implementação de programas de educação em saúde que permitam aos portadores de HAS conhecerem mais de sua patologia e consequentemente que os façam valorizar as proposições terapêuticas. Os enfermeiros são os profissionais responsáveis por lidar diretamente com o paciente e seus familiares, constituindo assim uma forma valiosa de garantia de adesão ao tratamento, reduzindo complicações.

\section{REFERENCIAS}

BALDisserA, V, D, A.; CARVAlHO, M, D, B.; PElloso, S, M. Adesão ao tratamento não-farmacológico entre hipertensos de um centro de saúde escola. Revista Gaúcha de Enfermagem. Porto Alegre - RS, 2009

Berlin JA, Colditz GA. A meta-analysis of physical activity in the prevention of coronary heart disease. Am J Epidemiol 1990; 132(4): 612-28. 40. Paffenbarger RS. Review: Con 
BRASIL. Ministério da Saúde. Secretaria de Atenção à Saúde. Departamento de Atenção Básica. Hipertensão arterial sistêmica. Brasília: Ministério da Saúde, 2006. 58 p. (Cadernos de Atenção Básica; 15). (Série A. Normas e Manuais Técnicos).

Chizzola PR, Mansur AJ, Luz PL, Bellotti G. Compliance with pharmacological treatment in outpatients from a brazilian cardiology referral center. São Paulo Med Journal. 1996; II $4: 1259-64$

Chobanian AV, Bakris GL, Black HR et al. Seventh Report of the Joint National Committee on Prevention, Detection, Evaluation, and Treatment of High Blood Pressure Hypertension 2003;42(6):1206-52.

European Heart Journal:doi/ı.1093/eurheartj/ehy339 2017 .

FERREIRA, C. Hipertensão Arterial. Emedix - Portal de saúde com informações sobre doenças. 2010. Disponível em. Acesso em 26/12/201I.

FORJAZ, C, L, M. Exercício resistido para o paciente hipertenso: indicação ou contraindicação. Revista Brasileira de Hipertensão. 2003.

GALVÃO,J.L. de; GINANI,G.F.;SILVA,G.V.da; ORTEGA，K.C.; MION JR., D. Adesão ao tratamento em hipertensão arterial sistólica isolada. Rev Bras Hipertes, v.ı,nı ,pag38-43, 2009. Disponivel em : http://departamentos.cardiol.br/dha/revista/I6-I/IIadesao.pdf Acesso em i5 out. 2019.

Horwitz RI, Horwitz SM. Adherence to the treatment and health outcomes. Arch Intern Med. 1993; 153:1863-8.

KIELlER, M; CUNHA, I.C.K.O. Assistência de enfermagem a pacientes com Hipertensão Arterial Sistêmica. Revista de Enfermagem UNISA. Disponível em:http://www.unisa.br/graduação/biológicas/enfer/revista/arquivos/2014-04.pdf Acesso em I5 out. 2019.

Krauss RM, Eckel RH; Howard BH et al. AHA Dietary Guidelines. Revision 2000: A statement for healthcare professionals from the Nutrition Committee of the American Heart Association. Stroke 2000; 31: 2751-66 
MacMahon S, Peto R, Cutler J. Blood pressure, stroke and coronary heart disease: effects of prolonged diferences in blood pressure-evidence from nine prospective observational studies corrected for dilution bias. Lancet 1995;335:765-74.

MacMahon S. Alcohol consumption and hypertension. Hypertension 1987; 9: II-I2I.

MENEZES, A. G. M. P.; GOBBI, D. Educação em saúde e Programa de Saúde da Família: atuação da enfermagem na prevenção de complicações em usuários hipertensos. Mundo da Saúde, São Paulo, v. 34, n. I, p. 97-102, 2010.

Minayo MCS. O desafio do conhecimento: pesquisa qualitativa em saúde. São Paulo: Hucitec; 1993. $269 \mathrm{p}$

MIO JR, D. Hipertensão Arterial. Sociedade Brasileira de Cardiologia e Sociedade Brasileira de Nefrologia. 2002.

Moreira, A.K. F,Santos,ZM. S.A, Caetano, J.A. Aplicação do modelo de crenças em saúde na adesão do trabalhador hipertenso ao tratamento. Physis, Rio de Janeiro, v.19,n.4,p.3,2009.

Motter FR, Teresa M, Olinto A, et al. Conhecimento sobre a farmacoterapia por portadores de Hipertensão Arterial Sistêmica. Ciênc Saúde Colet [internet]. 2013 I8(8):2263-2274. Disponível em: http://www.scielo.br/pdf/csc/vi8n8/ro.pdf. Acesso em is out. 2019

Oigman W. Bases hemodinâmicas da hipertensão arterial. Arq. bras. Cardiol.,49:303-8, 1987.

OLIVEIRA, A. Tratamento não medicamentoso da hipertensão arterial. Revista Bioquímica da Hipertensão. São Paulo - SP, 2011. Disponível em . Acesso em 25/10/2019.

Pierin, A.M; Mion Jr, D.; Fukushima, J.T; Pinto, A.R; Kaminaga, M.M. O perfil de um grupo de pessoas hipertensas de acordo com conhecimento e gravidade da doença. Rev. Esc. Enf. USP. 200I; 35(I): II-8.

Pinotti S, Mantovani MF, Giacomozzi LM. Percepção sobre a hipertensão arterial e qualidade de vida: contribuição para o cuidado de enfermagem. Cogitare Enferm. 2008;13(4): 526-34 
Pires, C.G.S.; Mussi, F. C. Crenças em saúde para o controle da hipertensão arterial. Ciência e Saúde Coletiva, Rio de Janeiro, v. I3, supl. 2, p. 2257-2267, 2008.

REINERS, Annelita; NOGUEIRA, Maria. Conscientização do usuário hipertenso para a adesão ao tratamento. Revista Latino-Am. Enfermagem. Disponível em:http://www.scielo.br/scielo.php?script=sci_arttext\&pid=SoIoII69200900o10ooro\&Ing= pt\&nrm=isoAcesso em is out. 2019.

Ribeiro AG, Cotta RMM, Silva LS, et al. Hipertensão arterial e orientação domiciliar: o papel estratégico da saúde da família. Rev Nutr [internet]. 2012 ; 25(2):271-282. Disponível em: http://www.scielo.br/scielo.php?script=sci_arttext \&pid=SI415-52732012000200009. Acesso em 2019 out is

Schroeder K, Fahey T, Ebrahim S. How can we improve adherence to blood pressurelowering medication in ambulatory care?: systematic review of randomized controlled trials. Arch Int Med. 2004; 164 (7):722-32

Secretaria do Estado de saúde (Paraná). Linha Guia. Hipertensão arterial. Secretaria saúde do Paraná, Curitiba, p. 26-26, 9 jul. 2014. Disponível em: http://www.saude.pr.gov.br/arquivos/File/web_final_hipertensao_linhaguia.pdf. Acesso em: 13 out. 2019

Smelter, S.C; Bare, B.G. Enfermagem medico-cirúrgica. ıo ed. Rio de Janeiro: Guanabara Koogan, 2005.

SMELTZER, S.C,; BARE,B.G. Tratado de Enfermagem Médico- Cirúrgico. Tradução José Eduardo Ferreira de figueiredo. Rio de Janeiro. Guanabara Konng, 2005. 437-445p. Acesso em I5 out. 2019.

Tavares NUL, Bertoldi AD, Thume $\mathrm{E}$ et al. Fatores associados a baixa adesão ao tratamento medicamentoso em idosos. Rev Saúde Pública [internet]. 2013 47(6):I092-IoI. Disponível em: http://www.scielo.br/scielo.php?script=sci_arttext\&pid=So03489102013000601092\&lng=en $\& \mathrm{nrm}=$ iso\&tlng=pt. Acesso em i5 out. 2019

Vitor AF, Monteiro FPM, Morais HCC, Vasconcelos JDP, Lopes MVO, Araújo TL. Perfil das condições de seguimento terapêutico em portadores de hipertensão arterial. Esc. Anna Nery Rev. Enferm. 2011; 15(2):251-260. 